\title{
New Absorption Bands and Isotopic Studies of Known Transitions in $\mathrm{CO}$
}

\author{
J. D. Simmons and S. G. Tilford* \\ Institute for Basic Standards, National Bureau of Standards, Washington, D.C. 20234
}

(April 21, 1971)

\begin{abstract}
Several new absorption bands of the $a^{\prime}{ }^{3} \Sigma^{+}-\mathrm{X}^{1} \Sigma^{+}$and $e^{3 \Sigma^{-}-\mathrm{X}^{1} \Sigma^{+}}$transitions in CO have been observed and analyzed. Vibrational levels of both the $a^{\prime}{ }^{3} \Sigma^{+}$and $e^{3} \Sigma^{-}$states are now known to within $0.4 \mathrm{eV}$ of their dissociation limits. Isotopic bands of ${ }^{13} \mathrm{C}^{16} \mathrm{O}$ in natural abundance have been analyzed for both transitions. The vibrational numbering of the $e^{3 \Sigma}$ state must be increased by one unit. New bands of the $\mathrm{I}^{1} \Sigma^{-}-\mathrm{X}^{1} \Sigma^{+}$and $\mathrm{D}^{1} \Delta-\mathrm{X}^{1} \Sigma^{+}$transitions are also reported.
\end{abstract}

Key words: Absorption spectrum; CO; electronic transitions; rotational analysis; vibrational analysis; vibrational and rotational constants.

\section{Introduction}

In this paper we report rotational analyses of several important new bands observed in the absorption spectrum of carbon monoxide. These analyses extend the observed data for four excited electronic states, $\mathrm{a}^{\prime}{ }^{3} \Sigma^{+}, e^{3} \Sigma^{-}, \mathrm{I}^{1} \Sigma^{-}$, and $\mathrm{D}^{1} \Delta$, which have previously been characterized in the literature.

Herzberg and Hugo [1] ${ }^{1}$ first characterized the forbidden $a^{\prime}{ }^{3} \Sigma^{+}-\mathrm{X}^{1} \Sigma^{+}$and $e^{3 \Sigma^{-}-\mathrm{X}^{1} \Sigma^{+}}$absorption systems of CO. For the $a^{\prime}-\mathrm{X}$ system they analyzed thirteen bands ranging from $v^{\prime}=2$ to 23 of the $a^{\prime}$ state. Krupenie [2] has pointed out the need for further experimental work to determine whether the upper state of the two emission bands at 2670 and $2980 A$, tentatively associated with the $f^{3} \Sigma^{+}-a^{3} \Pi$ transition, should be interpreted in terms of low vibrational levels of a separate electronic state, $f^{3} \Sigma^{+}$, or interpreted in terms of high vibrational levels, namely $v=31$ and 35 of the $a^{\prime}{ }^{3} \Sigma^{+}$state as originally proposed by Stepanov [3]. One objective in this work was to try to extend the observations of the $a^{\prime}$ state to higher vibrational levels beyond the $v=23$ level observed earlier. Although we observed five new $a^{\prime}-\mathrm{X}$ bands terminating on low vibrational levels of the $a^{\prime}$ state, only one new band was observed terminating on higher vibrational levels. The latter band at $87428.4 \mathrm{~cm}^{-1}$ has been assigned as $a^{\prime}-\mathrm{X}(38-0)$. Based on this assignment, arguments will be made in favor of Stepanov's assignment of the emission bands to the $a^{\prime}{ }^{3} \Sigma+-a^{3} \Pi$ transition. Herzberg and Hugo [1] analyzed six bands of the $e-\mathrm{X}$ system ranging from $v^{\prime}=2$ to 10 . We have analyzed eleven additional bands of this system that extend the obser-

*E. O. Hulburt Center for Space Research, Naval Research Laboratory, Washington. D.C. 20390.

${ }^{1}$ Figures in brackets indicate the literature references at the end of this paper. vations to $v^{\prime}=27$. One of the new bands was on the long wavelength side of the bands observed earlier, indicating that the vibrational numbering of the $e$ state should be increased by one unit. All statements concerning the $e$ state in this paper have taken that numbering change into account.

The new vibrational numbering of the $e$ state and the original numbering of the $a^{\prime}$ state were both confirmed by the analyses of ${ }^{13} \mathrm{C}^{16} \mathrm{O}$ isotopic bands.

Previous papers have characterized the forbidden $\mathrm{I}^{1} \Sigma^{-}-\mathrm{X}^{1} \Sigma^{+}$[4] and $\mathrm{D}^{1} \Delta-\mathrm{X}^{1} \Sigma^{+}$[5] absorption systems. We report here the analyses of two new bands of the $I-X$ system and one new band of the $D-X$ systems. These new bands involve transitions to high vibrational levels of the I and D states.

Revised vibrational and rotational coefficients and equilibrium constants have been determined for the $a^{\prime}, e, \mathrm{I}$, and $\mathrm{D}$ states by combining band origins and $B_{v}$-values from the earlier studies with similar data from the new analyses given here.

\section{Experimental Procedure}

Two sets of high-resolution absorption plates were used in the analyses. One set was taken at the National Research Council, Ottawa, Canada. The region 1550 to $1220 \AA$ was photographed in the seventh, eighth, and ninth orders of the $10-\mathrm{m}$ vacuum spectrograph described by Douglas and Potter [6]. The continuum light source was a Lyman discharge through argon. Overlapping orders were separated by a lithium fluoride prism-cylindrical lens combination described by Brix and Herzberg [7].

The second set of plates was taken at the U.S. Naval Research Laboratory. The region 1800 to $1060 \AA$ was photographed in the third, fourth, and fifth orders of a 
6.6-m vacuum spectrograph. The reciprocal dispersion was $0.42,0.32$, and $0.25 \mathrm{~A} / \mathrm{mm}$ in the third, fourth, and fifth orders, respectively. Microwave excited rare gas lamps were used as sources of background continua. No auxiliary means of order separation was used other than the limited range of the rare gas continua $\left(\mathrm{Xe}_{2}\right.$, 1800 to $1525 \AA ; \mathrm{Kr}_{2}, 1600$ to $1250 \AA$; $\mathrm{Ar}_{2}, 1260$ to 1060 $\AA$; (see Wilkinson and Byram [8])).

The strong Schumann-Runge absorption bands of the $\mathrm{O}_{2}$ molecule occur in the same region of the spectrum as the first few weak bands on the long wavelength end of the $a^{\prime 3} \Sigma^{+}-\mathrm{X}^{1} \Sigma^{+}$system of CO. To remove traces of molecular oxygen, the $\mathrm{CO}$ gas obtained from Matheson was passed over a hot zinc trap directly into the tank of the spectrograph which served as the absorption cell with a path length of $12 \mathrm{~m}$.

Pressures up to $6 \times 10^{3} \mathrm{~N} / \mathrm{m}^{2}$ were required to photograph the weakest bands of these systems. Wavelength reduction and band analyses were performed by techniques that have been described earlier $[4,9]$.

\section{3. $\mathbf{a}^{\prime 3} \Sigma^{+}-\mathbf{X}^{1} \Sigma^{+}$System}

The rotational structure of the $a^{\prime}-\mathrm{X}$ bands consists of four branches; two closely spaced R-form branches $\left({ }^{\mathrm{R}} \mathrm{R}\right.$ and $\left.{ }^{\mathrm{R}} \mathrm{Q}\right)$ and two closely spaced P-form branches $\left({ }^{\mathrm{P} P}\right.$ and $\left.{ }^{\mathrm{P}} \mathrm{Q}\right)$. The appearance of the bands is well illustrated by the spectrogram of the $5-0$ band at $1634.2 \AA$ reproduced in figure 2 of ref. [1] by Herzberg and Hugo.

We have been able to measure and analyze six new bands of this system that were not accessible on the earlier plates analyzed by Herzberg and Hugo. The analyses of these bands, for which $v^{\prime}=0,1,8,12,14$, and 38 , are given in table 1 . The vibrational assignment of the band at $87428.4 \mathrm{~cm}^{-1}$ as $(38-0)$ is somewhat ambiguous since it requires a very long extrapolation from $v^{\prime}=23$. When the band origins available between $v^{\prime}=0$ and 23 are fit to a 4 th order expression in $(v+1 / 2)$, the $(38-0)$ band is predicted at $87473 \mathrm{~cm}^{-1}$. Since the difference between the observed and calculated positions is only 10 percent of the vibrational spacing at this level, the above assignment is the most reasonable one.

The band origins $\nu_{0}$ and $B_{v}^{\prime}$-values for the $a^{\prime}-\mathrm{X}$ system are given in table 2 . Six of the bands reported by Herzberg and Hugo have been remeasured on the plates used in this study. The $\nu_{0}$ and $B_{v}^{\prime}$-values determined from these analyses have been included in table 2. Our results agree well with those of Herzberg and Hugo within the estimated experimental uncertainty.

Table 3 contains the revised vibrational and rotational constants for the $a^{\prime}$ state. The methods used to determine these constants and the reasons for including two separate sets are discussed in the last section of the paper. The analyses of the $(0-0)$ and $(1-0)$ bands have led to improved equilibrium constants.

Four $a^{\prime}-\mathrm{X}$ bands of ${ }^{13} \mathrm{C}^{16} \mathrm{O}$ have been observed in natural abundance with sufficient intensity to be measured, viz, (4-0), (5-0), (6-0), and (7-0). The rotational analyses of these bands are given in table 4 . The observed isotopic band origin shifts of 90.0, 113.9, 134.5, and $156.0 \mathrm{~cm}^{-1}$ for the $v^{\prime}=4$ to 7 levels, respectively, agree well with the corresponding shifts of 90.0, 112.9,
134.9 , and $156.1 \mathrm{~cm}^{-1}$ calculated from the constants reported in table 3 . Similarly, the observed $B_{v}^{\prime}$-values of $1.2107,1.1981,1.1743$, and 1.1638 for the isotopic bands agree reasonably well with the corresponding calculated values of $1.2106,1.1949,1.1794$, and 1.1640 $\mathrm{cm}^{-1}$, respectively. These observations confirm the vibrational numbering of the $a^{\prime}$ state given by Herzberg and Hugo [1].

The six perturbations between the $a^{\prime} 3 \Sigma^{+}$state and the $A^{1} \Pi$ state, for $J$-values accessible in absorption, have been described by Simmons, Bass, and Tilford [9]. It is of interest to note that the perturbation between $a^{\prime}, v=14$ and the A, $v=4$ levels occurs in the vicinity of $J=0$. The derived $a^{\prime}-X(14-0)$ band origin is shifted by a small amount toward lower energy and the rotational constant $\mathrm{B}_{14}^{\prime}$ is larger than the calculated value (see table 2). This perturbation is similar to the one between $\mathrm{A}^{1} \Pi, v=1$ and $d^{3} \Delta_{i}$, $v=5[9,10]$. Therefore, we suspect that emission from the $v=14$ level of the $a^{\prime}$ state should also be observed in the perturbation spectrum of $\mathrm{CO}$ (see Slanger and Black [11]).

Gerö [12] and Stepanov [3] have observed and analyzed the two CO emission bands at 2670 and $2980 \AA$ A. These authors proposed that the bands were part of the $a^{\prime} 3 \Sigma^{+}-a{ }^{3} \Pi$ transition involving high vibrational levels of the $a^{\prime}: \Sigma+$ state. Garg [13] and Gaydon [14] considered this interpretation doubtful and proposed instead that the bands should probably be assigned to a new transition, tentatively designated $f^{3} \Sigma^{+}-a^{3}$ II by Krupenie [2].

The analysis of the (38-0) $a^{\prime}-X \bar{b}$ and fixes a band origin and $B_{v}^{\prime}$-value for a high vibrational level of the $a^{\prime}$ state rather more accurately than did the earlier studies of perturbation data. It is, therefore, interesting to re-examine the merit of the earlier interpretation for the emission bands in the light of these new data. The upper states of the two emission bands are strongly perturbed by $v=0$ and $v=1$ levels of the $b^{3} \mathrm{E}^{+}$state. Ger̈ [12] and Stepanov [3] have performed separate rotational analyses on Gerö's experimental data using different techniques to deperturb the $B_{r}^{\prime}$-values and term values. The term values are given with respect to $X^{1} \Sigma^{+}, v=0, J=0$. Assuming the vibrational numbering of Stepanov and correcting the term values by $+36 \mathrm{~cm}^{-1}$ to account for revised $a-X$ data according to Krupenie, Gero's analysis gave the values:

$$
\begin{array}{ll}
B_{31}=0.83 \mathrm{~cm}^{-1} & B_{335}=0.75 \mathrm{~cm}^{-1} \\
E_{31}=83744 \mathrm{~cm}^{-1} & E_{35}=85969 \mathrm{~cm}^{-1} .
\end{array}
$$

Stepanov's analysis, which differed from Gerö's, gave:

$$
\begin{array}{ll}
B_{31}=0.800 \mathrm{~cm}^{-1} & B_{35}=0.711 \mathrm{~cm}^{-1} \\
E_{31}=83755 \mathrm{~cm}^{-1} & E_{35}=85960 \mathrm{~cm}^{-1} .
\end{array}
$$

These values agree reasonably well with the interpolated values of the same quantities obtained in this 
TABLE 1. Observed wave numbers in the $\mathrm{a}^{\prime}{ }^{3} \Sigma^{+}-\mathrm{X}^{1} \Sigma^{+}$system of $\mathrm{CO}$

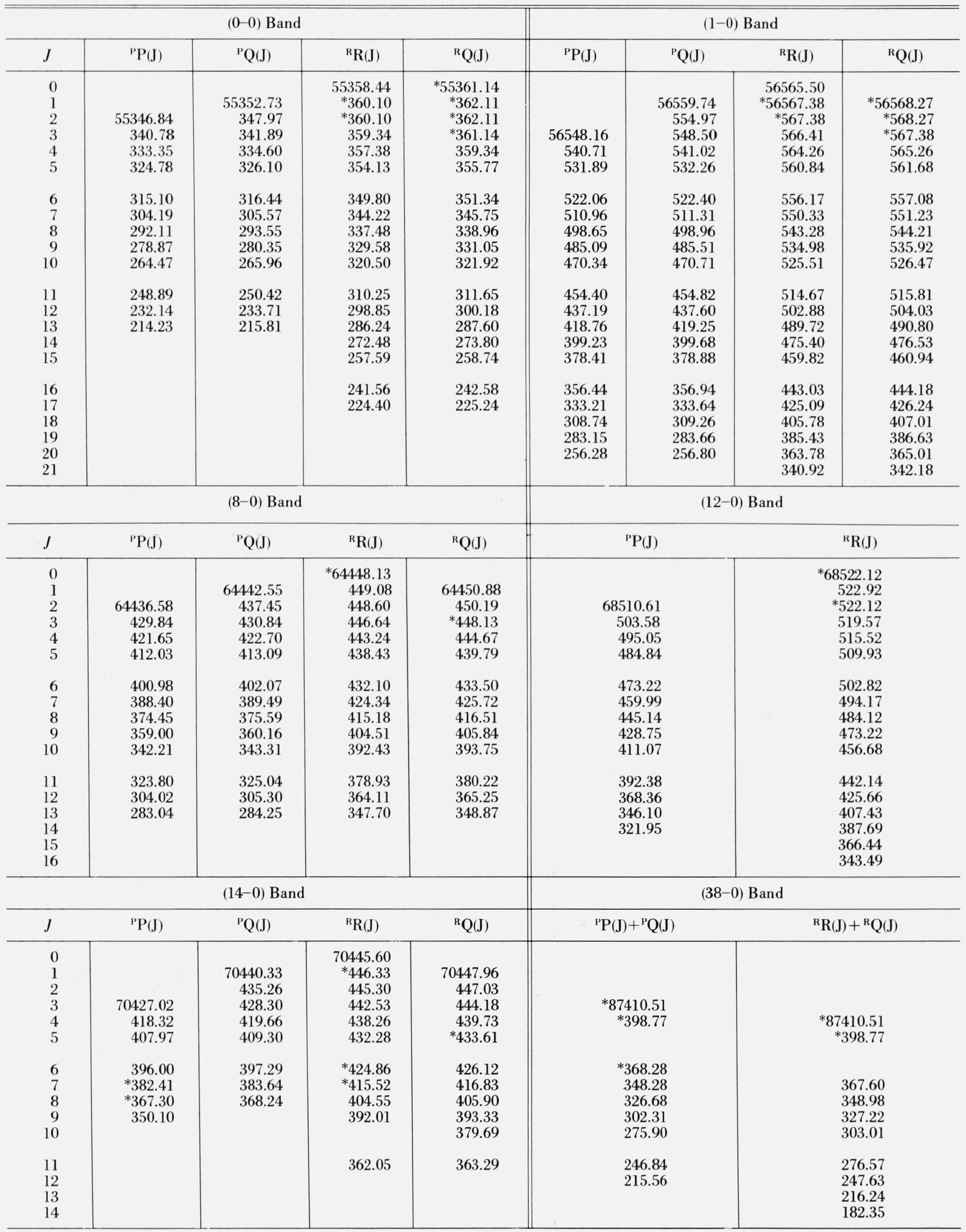


TABLE 2. Band origins and B-values for the $\mathrm{a}^{\prime} \cdot 3 \Sigma^{+}-\mathrm{X}^{1} \Sigma^{+}$system

\begin{tabular}{|c|c|c|c|c|c|}
\hline$v^{\prime}$ & $\begin{array}{c}\nu_{g}(\text { obs }) \\
\mathrm{cm}^{-1}\end{array}$ & $\begin{array}{l}\nu_{o}\left(\mathrm{calc}^{\mathrm{f}}{ }^{\mathrm{f}}\right. \\
\left(\mathrm{cm}^{-1}\right.\end{array}$ & $\begin{array}{c}B_{r}^{\prime}(\mathrm{obs}) \\
\mathrm{cm}^{-1}\end{array}$ & $\begin{array}{c}B_{r}^{\prime}(\text { calc })^{\mathrm{f}} \\
\mathrm{cm}^{-1}\end{array}$ & Footnotes \\
\hline 0 & 55355.75 & 55355.58 & 1.3350 & 1.3352 & (a) \\
\hline 1 & 56563.26 & 56563.30 & 1.3165 & 1.3169 & (a) \\
\hline 2 & 57750.16 & 57750.22 & 1.2995 & 1.2991 & (b) \\
\hline 3 & 58916.49 & 58916.51 & 1.2823 & 1.2818 & (b) \\
\hline 4 & 60062.64 & 60062.36 & 1.2658 & 1.2647 & (b) \\
\hline 5 & 61187.95 & 61187.98 & 1.2480 & 1.2479 & (") \\
\hline 6 & 62293.64 & 62293.59 & 1.2307 & 1.2313 & (b) \\
\hline 7 & 63379.44 & 63379.42 & 1.2143 & 1.2149 & (c) \\
\hline 8 & 64445.71 & 64445.72 & 1.1993 & 1.1985 & (a) \\
\hline 9 & 65492.53 & 65492.70 & 1.1813 & 1.1823 & (c) \\
\hline 10 & 66520.49 & 66520.57 & 1.1649 & 1.1661 & (c) \\
\hline 11 & & 67529.52 & & 1.1499 & \\
\hline 12 & ${ }^{e}(68520.0)$ & 68519.70 & $"(1.131)$ & 1.1338 & (a) \\
\hline 13 & 69491.36 & 69491.26 & 1.1171 & 1.1176 & (c) \\
\hline 14 & " 70443.55 & 70444.28 & 1.1053 & 1.1015 & (ii) \\
\hline 15 & & 71378.82 & & 1.0855 & \\
\hline 16 & 72295.04 & 72294.92 & 1.0691 & 1.0694 & $\left({ }^{(c)}\right.$ \\
\hline 17 & & 73192.56 & & 1.0533 & \\
\hline 18 & & 74071.67 & & 1.0373 & \\
\hline 19 & 74932.04 & 74932.17 & 1.0206 & 1.0213 & (b) \\
\hline 20 & 75773.91 & 75773.91 & 1.0037 & 1.0053 & (i) \\
\hline 21 & & 76596.73 & & 0.9892 & \\
\hline 22 & & 77400.42 & & .9732 & \\
\hline 23 & 78184.77 & 78184.74 & 0.9580 & .9570 & (b) \\
\hline 24 & & 78949.42 & & .9408 & \\
\hline 25 & & 79694.18 & & .9245 & \\
\hline 26 & & 80418.69 & & .9080 & \\
\hline 27 & & 81122.64 & & .8911 & \\
\hline 28 & & 81805.71 & & .8740 & \\
\hline 29 & & 82467.56 & & .8565 & \\
\hline 30 & & 83107.88 & & .8384 & \\
\hline 31 & 83744 & 83726.37 & .83 & .8196 & (d.e $)$ \\
\hline 32 & 83755 & 8120077 & .80 & & \\
\hline 33 & & 84896.84 & & .8001 & \\
\hline 34 & & 85448.41 & & .7579 & \\
\hline 35 & $\begin{array}{l}85969 \\
85960\end{array}$ & 85977.35 & $\begin{array}{l}.75 \\
.711\end{array}$ & .7349 & $(\mathrm{~d}, \mathrm{e})$ \\
\hline 36 & & 86483.62 & & .7104 & \\
\hline 37 & & 86967.26 & & .6840 & \\
\hline 38 & 87428.42 & 87428.42 & .6556 & .6556 & (a) \\
\hline
\end{tabular}

"Data from transition- reported in this paper.

${ }^{b}$ Data from Herzberg and Hugo [1].

c Data for upper state levels of transitions for which line assignments were reported by Herzberg and Hugo [1]. The constants and band origins given here are from measurements and analy ses of new hioh resolution plates.

"Term values and $B$-values given by Krupenie $|2|$ for the upper states of the emission bands at $2670 \quad 1$ and 29801

Band origins and $B_{r}^{\prime}$-values not included in the least squares fit.

$\nu(c a l c)$ and $B_{r}^{\prime}($ cealc) were calculated from polynomials for which the coefficients are niven in column 1 of table 3 .

study by using the high order vibrational and rotational coefficients given in the left column of table 3, namely:

$$
\begin{array}{ll}
B_{31}=0.8186 \mathrm{~cm}^{-1} & B_{35}=0.7349 \mathrm{~cm}^{-1} \\
E_{31}=83726 \mathrm{~cm}^{-1} & E_{35}=85977 \mathrm{~cm}^{-1} .
\end{array}
$$

Also, if one allows for the strong interactions with the $b$ state, the observed triplet splittings reported by Gerö are consistent with the splittings of other $a^{\prime}$ levels. From the above considerations, it does not seem necessary to invoke a separate electronic state other than $a^{\prime}: \Sigma^{+}$to explain the emission bands.

\section{The e ${ }^{3} \Sigma^{-}-X^{1} \Sigma^{+}$System}

The rotational structure of the $e-\mathrm{X}$ bands consists of five branches; ${ }^{S} \mathrm{R},{ }^{O} \mathrm{P},{ }^{\mathrm{Q}} \mathrm{Q},{ }^{\mathrm{Q}} \mathrm{R}$, and ${ }^{\mathrm{Q}} \mathrm{P}$. The appearance of these bands is illustrated by the spectrogram of the (4-0) band at $1471.0 \AA$ reproduced in figure 3 of reference [1] by Herzberg and Hugo. The Q-form branches lie very close together and the ${ }^{Q} R$ and ${ }^{Q} P$ branches are not resolved.

Herzberg and Hugo [1] observed four $e$-X bands, $v^{\prime}=2,3,4$, and 10, and unresolved Q-form branches for two others, $v^{\prime}=6$ and 8 . We have observed and analyzed eleven additional bands of this system. The assignments for these new bands plus a more extensive listing of the previously reported (6-0) band are given in table 5 . As in the case of the $a^{\prime}-\mathrm{X}$ system, we have also remeasured the $(2-0),(3-0)$, and $(4-0)$ bands of the $e-X$ system from the present plates. Band origins and $B_{r}^{\prime}$-values for all the observed vibrational levels are tabulated in table 6 . New spectroscopic constants have been determined for the $e$ state to take into account both the new data observed and the change of vibrational numbering of the $e$ state (see below). These constants are given in table 7 .

The analysis of a ${ }^{13} \mathrm{C}^{16} \mathrm{O} e-\mathrm{X}$ band observed in natural abundance is given in table 8 . The band origin and $B_{r}^{\prime}$-value for this band are $65842.43 \mathrm{~cm}^{-1}$ and $1.1838 \mathrm{~cm}^{-1}$, respectively. The band origin for the corresponding ${ }^{12} \mathrm{C}^{16} \mathrm{O}$ band is $65877.91 \mathrm{~cm}^{-1}$. The observed isotope shift for the band origin of $35.5 \mathrm{~cm}^{-1}$ agrees well with the calculated shift of $35.4 \mathrm{~cm}^{-1}$ if both bands are assumed to be $(2-0)$ bands. If, on the other hand, the bands are assumed to be (1-0) or (3-0) bands, and the spectroscopic constants are changed accordingly, isotopic shifts in the band origins of 11.6 and $59.2 \mathrm{~cm}^{-1}$ are predicted, which differ markedly from the observed value. Similar calculations for the isotope shift in the $B_{r}^{\prime}$-value also lead to the conclusion that the bands in question are the $(2-0)$ bands. These results, along with the fact that we have observed one new band on the long wavelength side of the bands reported earlier, indicate that the vibrational numbering for the $e^{3 \Sigma^{-}-}$state reported by Herzberg and Hugo [1] must be increased by one unit.

Perturbations between the $e^{3 \Sigma^{-}}$and $\mathrm{A}$ ' $I I$ states have been reported earlier [9]. It should be noted that the interaction between $e^{3} \Sigma^{-}, v=1$ and $\mathrm{A}^{1} \Pi, v=0$ is particularly strong. In fact, the intensity of the entire (1-0) $e-X$ band is much greater than for any other band in this system. Therefore, this perturbation will surely play a significant role in internal energy transfer processes in CO.

\section{The $I^{1} \Sigma^{-}-X^{1} \Sigma^{+}$and $D^{1} \Delta-X^{1} \Sigma^{+}$Systems}

The forbidden $\mathrm{I}-\mathrm{X}$ and $\mathrm{D}-\mathrm{X}$ transitions have been characterized in two earlier papers $[4,5]$ of the present investigation. Both systems are observed in absorption 
TABLE 3. Vibrational and rotational constants for $\mid$ the $\mathrm{a}^{\prime}{ }^{3} \Sigma^{+}$state $^{\mathrm{a}}$

\begin{tabular}{l|r}
\hline \hline & \\
$Y_{01}^{(5)}=1.3446 \pm 0.0016 \mathrm{~cm}^{-1}$ & $B_{e} \cong Y_{01}^{(1,2)}=1.3430 \pm 0.0012 \mathrm{~cm}^{-1}$ \\
$Y_{11}^{(5)}=(-1.8921 \pm 0.1163) \times 10^{-2} \mathrm{~cm}^{-1}$ & $-\alpha_{e} \cong Y_{11}^{(1,2)}=-0.0173 \pm 0.0007 \mathrm{~cm}^{-1}$ \\
$Y_{21}^{(5)}=(3.4542 \pm 2.4550) \times 10^{-4} \mathrm{~cm}^{-1}$ & $T_{00}=55355.75 \pm 0.30 \mathrm{~cm}^{-1}$ \\
$Y_{31}^{(5)}=(-2.2037 \pm 2.0532) \times 10^{-5} \mathrm{~cm}^{-1}$ & $T_{e}=55826.0 \pm 1.8 \mathrm{~cm}^{-1}$ \\
$Y_{41}^{(5)}=(7.2205 \pm 7.1937) \times 10^{-7} \mathrm{~cm}^{-1}$ & $\omega_{e} \cong Y_{10}^{(2,3)}=1227.8 \pm 1.1 \mathrm{~cm}^{-1}$ \\
$Y_{51}^{(5)}=(-9.4737 \pm 8.6378) \times 10^{-9} \mathrm{~cm}^{-1}$ & $\left.-x \omega_{e} \cong Y_{20}^{(2,}, 3\right)=-10.22 \pm 0.38 \mathrm{~cm}^{-1}$ \\
$T_{e 0}^{(6)}=54743.90 \pm 0.30 \mathrm{~cm}^{-1}$ & \\
$T_{e}^{(6)}=55825.49 \pm 0.30 \mathrm{~cm}^{-1}$ & \\
$Y_{10}^{(6)}=1228.604 \pm 0.230 \mathrm{~cm}^{-1}$ & \\
$Y_{2(6)}^{(6)}=-10.4675 \pm 0.0710 \mathrm{~cm}^{-1}$ & $I_{e}=20.842 \times 10^{-40} \mathrm{~g} \mathrm{~cm}^{2}$ \\
$Y_{30}^{(6)}=(9.0719 \pm 9.3079) \times 10^{-3} \mathrm{~cm}^{-1}$ & \\
$Y_{40}^{(6)}=(2.5910 \pm 0.5757) \times 10^{-3} \mathrm{~cm}^{-1}$ & \\
$Y_{50}^{(6)}=(-1.0168 \pm 0.1646) \times 10^{-4} \mathrm{~cm}^{-1}$ & \\
$Y_{60}^{(6)}=(1.0100 \pm 0.1727) \times 10^{-6} \mathrm{~cm}^{-1}$ & \\
&
\end{tabular}

a Note that two sets of rotational and vibrational constants are given in the table. See the section of the text entitled, "Determination of vibrational and rotational constants" for a dis

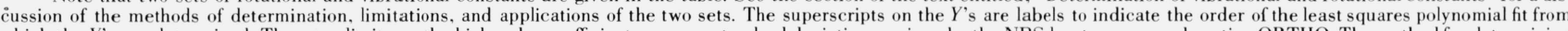

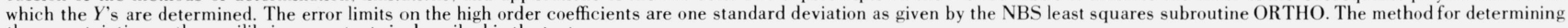
the uncertainties on the equilibrium constants is described in the text.

${ }^{b}$ Constant $D$-value used to determine $B_{r}$-values.

TABLE 4. Observed wave numbers in the $\mathrm{a}^{\prime}{ }^{3} \Sigma^{+}-\mathrm{X}^{1} \Sigma^{+}$system of ${ }^{13} \mathrm{C}^{16} \mathrm{O}$

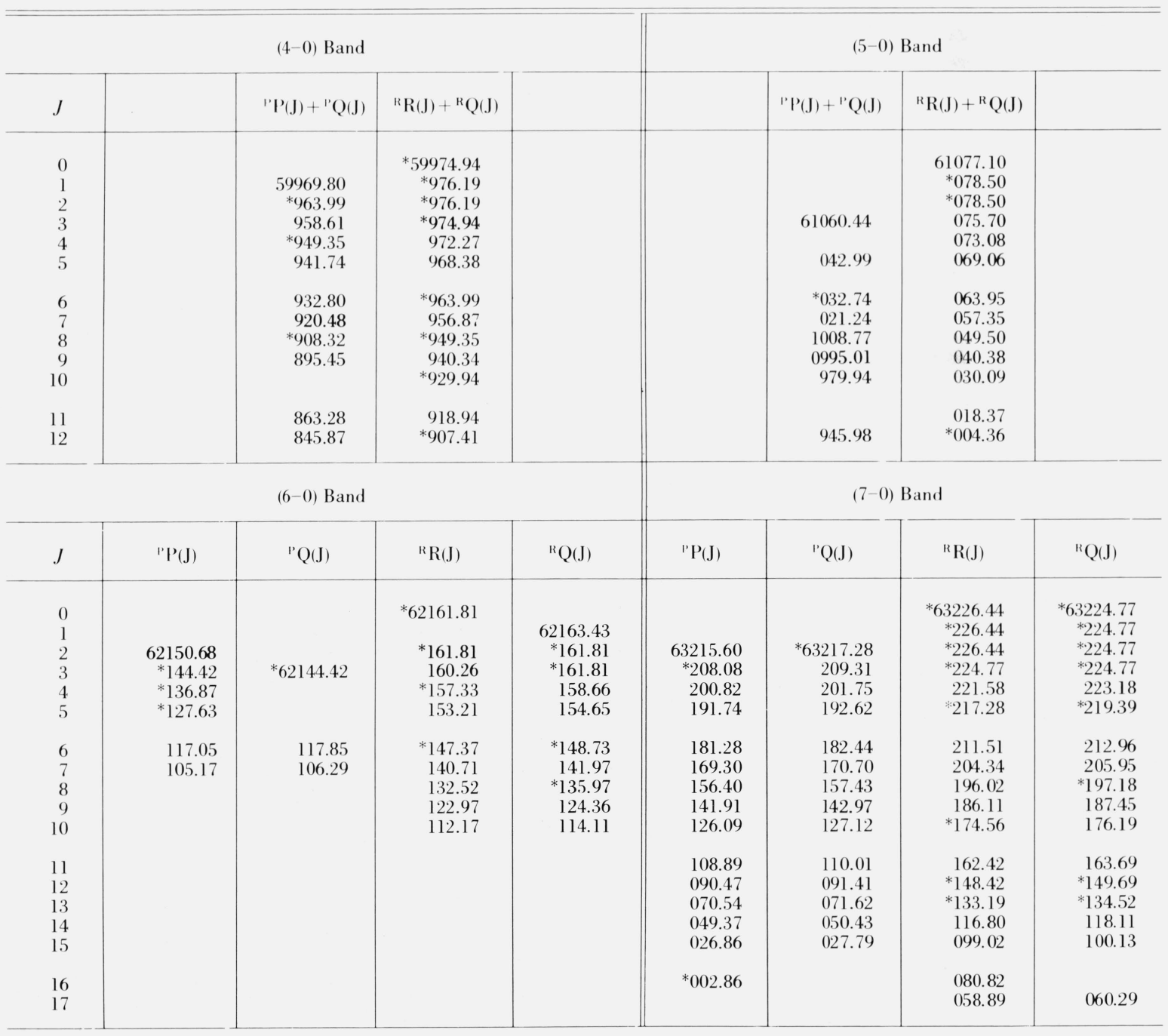

*Blended lines. 
TABLE 5. Observed wave numbers in the $\mathrm{e}^{3 \Sigma^{-}-\mathrm{X}^{1} \Sigma^{+}}$system of $\mathrm{CO}$

\begin{tabular}{|c|c|c|c|c|c|c|c|c|c|}
\hline \multirow[b]{2}{*}{$J$} & \multirow[b]{2}{*}{${ }^{\circ} \mathrm{P}(\mathrm{J})$} & \multirow{2}{*}{$\begin{array}{c}(0-0) \text { Band } \\
{ }^{Q} Q(J)\end{array}$} & \multirow[b]{2}{*}{${ }^{Q} P(J)+{ }^{Q} R(J)$} & \multirow[b]{2}{*}{${ }^{\mathrm{s}} \mathrm{R}(\mathrm{J})$} & \multicolumn{5}{|c|}{$\mid 1-0)$ Band } \\
\hline & & & & & ${ }^{\circ} \mathrm{P}(\mathrm{J})$ & ${ }^{\mathrm{Q}} \mathrm{P}(\mathrm{J})$ & ${ }^{\mathrm{Q}} \mathrm{Q}(\mathrm{J})$ & ${ }^{\mathrm{Q}} \mathrm{R}(\mathrm{J})$ & ${ }^{\mathrm{s}} \mathrm{R}(\mathrm{J})$ \\
\hline $\begin{array}{l}0 \\
1 \\
2 \\
3 \\
4 \\
5\end{array}$ & $\begin{array}{r}63692.83 \\
683.83 \\
673.52 \\
661.92\end{array}$ & $\begin{array}{r}63703.50 \\
700.94 \\
697.05 \\
691.86 \\
685.32\end{array}$ & $\begin{array}{r}63704.45 \\
703.23 \\
700.54 \\
696.61 \\
691.41 \\
684.89\end{array}$ & $\begin{array}{r}63711.80 \\
715.66 \\
* 718.14 \\
* 719.45 \\
* 719.45 \\
* 718.14\end{array}$ & $\begin{array}{r}64970.17 \\
* 781.33 \\
770.89 \\
759.13\end{array}$ & $\begin{array}{r}64797.31 \\
793.33 \\
788.10 \\
* 781.33\end{array}$ & $\begin{array}{r}* 64801.43 \\
798.76 \\
794.76 \\
789.56 \\
783.01\end{array}$ & $\begin{array}{r}64801.78 \\
800.38 \\
797.77 \\
793.84 \\
788.65 \\
782.35\end{array}$ & $\begin{array}{r}64808.81 \\
812.54 \\
814.96 \\
816.01 \\
815.85 \\
814.14\end{array}$ \\
\hline $\begin{array}{r}6 \\
7 \\
8 \\
9 \\
10\end{array}$ & $\begin{array}{l}649.11 \\
634.88 \\
619.11 \\
584.44\end{array}$ & $\begin{array}{l}677.56 \\
668.54 \\
658.10 \\
646.49 \\
633.55\end{array}$ & $\begin{array}{l}677.07 \\
668.01 \\
657.63 \\
645.95 \\
632.95\end{array}$ & $\begin{array}{l}715.41 \\
711.43 \\
706.00 \\
699.72 \\
691.63\end{array}$ & $\begin{array}{r}746.51 \\
732.39 \\
* 717.24 \\
* 701.20 \\
* 685.31\end{array}$ & $\begin{array}{r}773.51 \\
764.12 \\
* 753.57 \\
741.63\end{array}$ & $\begin{array}{l}* 775.22 \\
* 766.21 \\
* 755.83 \\
* 744.24 \\
* 731.75\end{array}$ & $\begin{array}{l}* 774.89 \\
* 766.43 \\
* 758.29 \\
* 737.53 \\
* 725.51\end{array}$ & $\begin{array}{r}811.18 \\
806.93 \\
* 801.43 \\
794.50 \\
786.23\end{array}$ \\
\hline $\begin{array}{l}11 \\
12 \\
13 \\
14 \\
15\end{array}$ & $\begin{array}{l}565.35 \\
544.64 \\
522.72 \\
499.60 \\
474.90\end{array}$ & $\begin{array}{r}619.34 \\
* 603.82 \\
* 586.65 \\
* 568.48 \\
* 548.89\end{array}$ & & $\begin{array}{l}682.31 \\
671.58 \\
\\
646.36\end{array}$ & $\begin{array}{r}* 656.83 \\
637.37\end{array}$ & $\begin{array}{r}713.90 \\
697.95 \\
680.86 \\
662.43 \\
*^{*} 642.93\end{array}$ & $\begin{array}{l}* 718.79 \\
* 705.67 \\
* 674.59 \\
* 658.07 \\
* 639.13\end{array}$ & & $\begin{array}{r}776.82 \\
* 766.21 \\
* 754.33 \\
* 741.48 \\
* 728.68\end{array}$ \\
\hline $\begin{array}{l}16 \\
17 \\
18 \\
19 \\
20 \\
21\end{array}$ & $\begin{array}{l}449.14 \\
421.94 \\
393.63\end{array}$ & $\begin{array}{l}* 528.35 \\
* 506.36 \\
* 482.91 \\
* 458.32 \\
* 432.52 \\
* 405.28\end{array}$ & & $\begin{array}{l}616.78 \\
600.03\end{array}$ & & $\begin{array}{l}* 622.49 \\
{ }^{*} 602.05 \\
{ }^{*} 569.62\end{array}$ & $\begin{array}{l}* 618.47 \\
* 596.25 \\
* 545.88\end{array}$ & & $\begin{array}{l}* 703.76 \\
* 687.95\end{array}$ \\
\hline
\end{tabular}


(6-0) Band

(7-0) Band

\begin{tabular}{|c|c|c|c|c|c|c|c|c|}
\hline \multicolumn{5}{|c|}{$(6-0)$ Band } & \multicolumn{4}{|c|}{$(7-0)$ Band } \\
\hline$J$ & ${ }^{o} \mathrm{P}(\mathrm{J})$ & ${ }^{\mathrm{Q}} \mathrm{Q}(\mathrm{J})$ & ${ }^{Q} P(J)+{ }^{Q} R(J)$ & ${ }^{\mathrm{s}} \mathrm{R}(\mathrm{J})$ & ${ }^{o} \mathrm{P}(\mathrm{J})$ & ${ }^{\mathrm{Q}} \mathrm{Q}(\mathrm{J})$ & ${ }^{Q} P(J)+{ }^{Q} R(J)$ & ${ }^{\mathrm{s}} \mathrm{R}(\mathrm{J})$ \\
\hline $\begin{array}{l}0 \\
1 \\
2 \\
3 \\
4 \\
5\end{array}$ & $\begin{array}{r}69974.71 \\
965.45 \\
* 954.67 \\
942.52\end{array}$ & $\begin{array}{r}69985.14 \\
982.14 \\
977.64 \\
971.62 \\
964.11\end{array}$ & $\begin{array}{r}69986.22 \\
984.78 \\
981.62 \\
977.10 \\
971.10 \\
963.58\end{array}$ & $\begin{array}{r}69992.95 \\
996.27 \\
* 998.00 \\
* 998.00 \\
996.72 \\
993.92\end{array}$ & $\begin{array}{r}* 70953.74 \\
* 944.45 \\
\quad * 933.57 \\
\quad * 921.41\end{array}$ & $\begin{array}{r}70964.37 \\
* 961.21 \\
956.64 \\
950.49 \\
942.81\end{array}$ & $\begin{array}{r}70965.26 \\
963.85 \\
960.58 \\
956.07 \\
949.90 \\
942.21\end{array}$ & $\begin{array}{r}* 70972.01 \\
* 975.12 \\
* 976.66 \\
* 976.66 \\
* 975.12 \\
* 972.01\end{array}$ \\
\hline $\begin{array}{r}6 \\
7 \\
8 \\
9 \\
10\end{array}$ & $\begin{array}{l}928.81 \\
913.59 \\
896.89 \\
878.68 \\
858.91\end{array}$ & $\begin{array}{r}954.99 \\
* 944.60 \\
932.56 \\
919.03 \\
904.01\end{array}$ & $\begin{array}{l}954.67 \\
944.03 \\
932.00 \\
918.44 \\
903.41\end{array}$ & $\begin{array}{l}989.59 \\
983.79 \\
976.45 \\
967.49 \\
957.14\end{array}$ & $\begin{array}{r}* 907.46 \\
892.10 \\
875.19 \\
856.74 \\
836.69\end{array}$ & $\begin{array}{l}933.57 \\
922.82 \\
910.54 \\
896.64 \\
881.32\end{array}$ & $\begin{array}{l}932.94 \\
922.19 \\
909.84 \\
895.93 \\
880.62\end{array}$ & $\begin{array}{r}967.49 \\
* 961.21 \\
* 953.74 \\
* 944.45 \\
* 933.57\end{array}$ \\
\hline $\begin{array}{l}11 \\
12 \\
13 \\
14\end{array}$ & & $\begin{array}{l}887.47 \\
869.47 \\
849.89\end{array}$ & $\begin{array}{l}886.88 \\
868.86 \\
849.40\end{array}$ & $* 944.60$ & $\begin{array}{l}815.25 \\
792.09\end{array}$ & $\begin{array}{l}864.39 \\
845.95 \\
825.97 \\
804.55\end{array}$ & $\begin{array}{l}863.71 \\
845.23 \\
825.27 \\
803.63\end{array}$ & $\begin{array}{l}* 921.41 \\
* 907.46\end{array}$ \\
\hline \multicolumn{5}{|c|}{$(12-0)$ Band } & \multicolumn{4}{|c|}{ (14-0) Band } \\
\hline$J$ & & ${ }^{\mathrm{Q}} \mathrm{Q}(\mathrm{J})$ & ${ }^{\mathrm{Q}} \mathrm{P}(\mathrm{J})+{ }^{\mathrm{Q}} \mathrm{R}(\mathrm{J})$ & ${ }^{\mathrm{s}} \mathrm{R}(\mathrm{J})$ & ${ }^{o} \mathrm{P}(\mathrm{J})$ & ${ }^{\mathrm{Q}} \mathrm{Q}(J)^{\mathrm{a}}$ & & ${ }^{\mathrm{s}} \mathrm{R}(\mathrm{J})$ \\
\hline $\begin{array}{l}0 \\
1 \\
2 \\
3 \\
4 \\
5\end{array}$ & & $\begin{array}{r}* 75581.49 \\
* 578.58 \\
573.03 \\
* 566.39 \\
557.85\end{array}$ & $\begin{array}{r}* 75581.49 \\
* 578.58 \\
572.52 \\
* 566.39 \\
557.29\end{array}$ & & $\begin{array}{r}77288.71 \\
279.04 \\
267.94 \\
254.67\end{array}$ & $\begin{array}{r}* 7300.37 \\
299.06 \\
295.19 \\
289.99 \\
282.85 \\
274.09\end{array}$ & & $\begin{array}{r}77308.94 \\
308.39 \\
305.20 \\
* 300.37\end{array}$ \\
\hline $\begin{array}{r}6 \\
7 \\
8 \\
9 \\
10\end{array}$ & & $\begin{array}{r}* 547.46 \\
535.67 \\
522.25\end{array}$ & $\begin{array}{r}* 547.46 \\
* 535.11 \\
521.28\end{array}$ & $\begin{array}{r}75562.07 \\
551.06\end{array}$ & $\begin{array}{l}240.13 \\
223.59 \\
205.30 \\
185.15\end{array}$ & $\begin{array}{r}* 263.67 \\
250.97 \\
237.19 \\
221.21 \\
203.17\end{array}$ & & $\begin{array}{r}294.36 \\
285.70 \\
275.87 \\
* 263.67 \\
250.62\end{array}$ \\
\hline $\begin{array}{l}11 \\
12 \\
13 \\
14 \\
15\end{array}$ & & & & & $\begin{array}{r}139.36 \\
114.50 \\
087.38 \\
058.50 \\
7027.95\end{array}$ & $\begin{array}{l}183.39 \\
161.94 \\
139.36 \\
114.50 \\
088.12\end{array}$ & & $\begin{array}{l}235.02 \\
218.05 \\
199.30 \\
179.22 \\
157.65\end{array}$ \\
\hline $\begin{array}{l}16 \\
17 \\
18\end{array}$ & & & & & 6995.59 & $\begin{array}{r}060.29 \\
7031.04 \\
6999.88\end{array}$ & & $\begin{array}{l}133.27 \\
108.07\end{array}$ \\
\hline
\end{tabular}

a All three Q-type branches unresolved.

*Blended lines. 
TABLE 5. Observed wave numbers in the $\mathrm{e}^{3} \Sigma^{-}-\mathrm{X}^{1} \Sigma^{+}{ }^{+}$system of $\mathrm{CO}-\mathrm{Continued}$

\begin{tabular}{|c|c|c|c|c|c|c|c|}
\hline \multicolumn{5}{|c|}{ (15-0) Band } & \multicolumn{3}{|c|}{ (17-0) Band } \\
\hline$J$ & ${ }^{o} \mathrm{P}(\mathrm{J})$ & ${ }^{\mathrm{Q}} \mathrm{Q}(\mathrm{J})$ & ${ }^{Q} \mathrm{P}(\mathrm{J})+{ }^{Q} \mathrm{R}(\mathrm{J})$ & ${ }^{\mathrm{s}} \mathrm{R}(\mathrm{J})$ & ${ }^{\circ} \mathrm{P}(\mathrm{J})$ & ${ }^{\mathrm{a}} \mathrm{Q}(\mathrm{J})^{\mathrm{a}}$ & ${ }^{\mathrm{s}} \mathrm{R}(\mathrm{J})$ \\
\hline $\begin{array}{l}0 \\
1 \\
2 \\
3 \\
4 \\
5\end{array}$ & $\begin{array}{r}78108.56 \\
097.22 \\
084.10\end{array}$ & $\begin{array}{r}78128.70 \\
124.92 \\
119.51 \\
112.30 \\
103.19\end{array}$ & $\begin{array}{r}78127.80 \\
124.12 \\
118.67 \\
111.50 \\
102.46\end{array}$ & $\begin{array}{r}78135.46 \\
137.83 \\
138.27 \\
137.01 \\
*^{*} 133.75 \\
128.89\end{array}$ & $\begin{array}{r}79720.50 \\
710.94 \\
699.41 \\
686.08\end{array}$ & $\begin{array}{r}79730.59 \\
726.94 \\
721.02 \\
713.66 \\
704.45\end{array}$ & $\begin{array}{r}* 79738.16 \\
* 739.92 \\
* 739.92 \\
* 738.16 \\
734.79 \\
729.13\end{array}$ \\
\hline $\begin{array}{r}6 \\
7 \\
8 \\
9 \\
10\end{array}$ & $\begin{array}{l}069.21 \\
052.48 \\
033.95\end{array}$ & $\begin{array}{r}092.47 \\
* 079.87 \\
065.34 \\
049.12 \\
031.10\end{array}$ & $\begin{array}{l}091.53 \\
078.99 \\
064.63 \\
048.31 \\
030.32\end{array}$ & $\begin{array}{l}122.20 \\
113.63 \\
103.31 \\
091.23 \\
077.13\end{array}$ & $\begin{array}{l}670.89 \\
653.80 \\
634.84 \\
514.11 \\
591.44\end{array}$ & $\begin{array}{l}693.06 \\
679.82 \\
664.92 \\
648.17 \\
629.57\end{array}$ & $\begin{array}{l}721.77 \\
713.01 \\
701.87 \\
688.96 \\
674.44\end{array}$ \\
\hline $\begin{array}{l}11 \\
12 \\
13 \\
14 \\
15\end{array}$ & & & & 061.31 & $\begin{array}{l}566.58 \\
540.51 \\
512.29 \\
482.03 \\
450.02\end{array}$ & $\begin{array}{l}608,78 \\
586.41 \\
561.87 \\
535.78 \\
507.69\end{array}$ & $\begin{array}{l}657.38 \\
638.68 \\
618.35 \\
596.12 \\
571.87\end{array}$ \\
\hline $\begin{array}{l}16 \\
17 \\
18 \\
19 \\
20\end{array}$ & & & & & $\begin{array}{l}416.13 \\
380.35 \\
342.70 \\
303.31\end{array}$ & $\begin{array}{l}477.72 \\
445.83 \\
412.10 \\
376.50 \\
338.97\end{array}$ & $\begin{array}{l}545.71 \\
517.79 \\
487.95 \\
456.19 \\
422.37\end{array}$ \\
\hline $\begin{array}{l}21 \\
22 \\
23\end{array}$ & & & & & 218.74 & $\begin{array}{l}299.58 \\
215.30\end{array}$ & $\begin{array}{l}387.45 \\
350.04\end{array}$ \\
\hline
\end{tabular}


(21-0) Band

(24-0) Band

\begin{tabular}{|c|c|c|c|c|c|c|}
\hline$J$ & ${ }^{o} \mathrm{P}(\mathrm{J})$ & ${ }^{\mathrm{Q}} \mathrm{Q}(\mathrm{J})^{\mathrm{a}}$ & ${ }^{\mathrm{s}} \mathrm{R}(\mathrm{J})$ & ${ }^{o} \mathrm{P}(\mathrm{J})$ & ${ }^{\mathrm{Q}} \mathrm{Q}(\mathrm{J}){ }^{\mathrm{a}}$ & ${ }^{\mathrm{s}} \mathrm{R}(\mathrm{J})$ \\
\hline $\begin{array}{l}0 \\
1 \\
2 \\
3 \\
4 \\
5\end{array}$ & $\begin{array}{r}* 82682.35 \\
672.67 \\
660.79 \\
646.85\end{array}$ & $\begin{array}{r}82692.41 \\
688.31 \\
* 682.35 \\
674.20 \\
664.13\end{array}$ & $\begin{array}{r}82699.11 \\
* 700.67 \\
* 700.67 \\
698.32 \\
693.69 \\
687.37\end{array}$ & $\begin{array}{r}* 84668.49 \\
* 659.51 \\
647.14 \\
632.97\end{array}$ & $\begin{array}{r}84679.01 \\
674.69 \\
*^{6} 668.49 \\
{ }^{*} 659.51 \\
{ }^{*} 649.14\end{array}$ & $\begin{array}{r}84685.64 \\
* 686.15 \\
* 686.15 \\
682.90 \\
677.74 \\
670.33\end{array}$ \\
\hline $\begin{array}{r}6 \\
7 \\
8 \\
9 \\
10\end{array}$ & $\begin{array}{l}631.45 \\
613.46 \\
593.83 \\
572.07 \\
548.15\end{array}$ & $\begin{array}{l}652.05 \\
637.89 \\
621.75 \\
603.10 \\
583.61\end{array}$ & $\begin{array}{l}678.75 \\
668.20 \\
655.74 \\
641.19 \\
624.74\end{array}$ & $\begin{array}{l}616.62 \\
598.34 \\
577.79 \\
555.14 \\
530.17\end{array}$ & $\begin{array}{r}* 635.86 \\
621.06 \\
604.01 \\
584.73 \\
563.33\end{array}$ & $\begin{array}{r}660.91 \\
* 649.14 \\
* 635.86 \\
619.79 \\
601.68\end{array}$ \\
\hline $\begin{array}{l}11 \\
12 \\
13 \\
14 \\
15\end{array}$ & $\begin{array}{l}522.40 \\
494.40 \\
464.44 \\
432.68 \\
398.97\end{array}$ & $\begin{array}{r}561.23 \\
537.08 \\
* 510.94 \\
* 482.89 \\
* 452.95\end{array}$ & $\begin{array}{r}606.21 \\
585.59 \\
562.87 \\
538.18 \\
* 510.94\end{array}$ & $\begin{array}{l}503.34 \\
474.25 \\
442.94 \\
409.62 \\
374.04\end{array}$ & $\begin{array}{l}539.73 \\
514.00 \\
486.22 \\
456.32 \\
424.00\end{array}$ & $\begin{array}{r}581.56 \\
* 558.64 \\
534.64 \\
507.89 \\
479.16\end{array}$ \\
\hline $\begin{array}{l}16 \\
17 \\
18\end{array}$ & & 420.07 & $\begin{array}{l}* 482.89 \\
* 452.95\end{array}$ & 336.48 & $\begin{array}{l}389.64 \\
353.16\end{array}$ & $\begin{array}{l}448.24 \\
415.16 \\
379.85\end{array}$ \\
\hline \multicolumn{4}{|c|}{$(25-0)$ Band } & \multicolumn{3}{|c|}{$(27-0)$ Band } \\
\hline$J$ & ${ }^{o} \mathbf{P}(\mathbf{J})$ & ${ }^{\mathrm{Q}} \mathrm{Q}(\mathrm{J})^{\mathrm{a}}$ & ${ }^{\mathrm{s}} \mathrm{R}(\mathrm{J})$ & ${ }^{o} \mathrm{P}(\mathrm{J})$ & ${ }^{\mathrm{Q}} \mathrm{Q}(\mathrm{J}){ }^{\mathrm{a}}$ & ${ }^{\mathrm{s}} \mathrm{R}(\mathrm{J})$ \\
\hline $\begin{array}{l}0 \\
1 \\
2 \\
3 \\
4 \\
5\end{array}$ & $\begin{array}{r}* 85270.52 \\
* 258.62 \\
* 244.23\end{array}$ & $\begin{array}{r}85290.52 \\
285.91 \\
279.70 \\
* 270.52 \\
259.52\end{array}$ & $\begin{array}{r}85295.04 \\
297.59 \\
296.71 \\
293.39 \\
288.07 \\
280.29\end{array}$ & $\begin{array}{r}86418.05 \\
* 407.65 \\
* 395.67 \\
381.97\end{array}$ & $\begin{array}{r}* 86423.37 \\
416.26 \\
* 407.65 \\
* 395.67\end{array}$ & $\begin{array}{r}* 86434.92 \\
* 434.92 \\
433.47 \\
429.56 \\
* 423.37 \\
415.34\end{array}$ \\
\hline $\begin{array}{r}6 \\
7 \\
8 \\
9 \\
10\end{array}$ & $\begin{array}{l}* 228.76 \\
* 209.20 \\
* 188.42 \\
* 165.39 \\
* 140.21\end{array}$ & $\begin{array}{r}246.59 \\
231.24 \\
* 213.57 \\
* 192.40 \\
172.30\end{array}$ & $\begin{array}{l}* 270.52 \\
* 258.62 \\
* 244.23 \\
* 228.76 \\
* 209.20\end{array}$ & $\begin{array}{l}364.20 \\
345.08 \\
323.63 \\
299.89 \\
273.88\end{array}$ & $\begin{array}{l}381.26 \\
365.48 \\
347.06 \\
327.00 \\
304.17\end{array}$ & $\begin{array}{l}404.59 \\
391.71 \\
376.43 \\
358.89 \\
339.12\end{array}$ \\
\hline $\begin{array}{l}11 \\
12 \\
13 \\
14 \\
15\end{array}$ & $\begin{array}{r}* 112.02 \\
* 051.73 \\
* 5017.43\end{array}$ & $\begin{array}{r}148.89 \\
122.98 \\
* 5093.28\end{array}$ & $\begin{array}{l}* 188.42 \\
* 165.39 \\
* 140.21 \\
* 112.02\end{array}$ & $\begin{array}{l}245.59 \\
215.04 \\
182.18 \\
147.13\end{array}$ & $\begin{array}{l}278.94 \\
251.43 \\
221.66 \\
189.46 \\
155.48\end{array}$ & $\begin{array}{l}292.47 \\
265.89 \\
236.77 \\
205.43\end{array}$ \\
\hline $\begin{array}{l}16 \\
17 \\
18 \\
19\end{array}$ & 4942.82 & $\begin{array}{r}4957.28 \\
917.88 \\
871.62\end{array}$ & $\begin{array}{l}* 051.73 \\
* 017.43\end{array}$ & & & $\begin{array}{l}171.74 \\
135.73\end{array}$ \\
\hline
\end{tabular}


TABLE 6. Band origins and $\mathrm{B}_{\mathrm{v}}^{\prime}$-values for the $\mathrm{e}^{3} \Sigma^{-}-\mathrm{X}^{1} \Sigma^{+}$system

\begin{tabular}{|c|c|c|c|c|c|}
\hline$v^{\prime}$ & $\begin{array}{c}\nu_{0}(\mathrm{obs}) \\
\mathrm{cm}^{-1}\end{array}$ & $\begin{array}{c}\nu_{o}(\text { calc }) \\
\mathrm{cm}^{-1}\end{array}$ & $\begin{array}{c}B_{r}^{\prime}(\mathrm{obs}) \\
\mathrm{cm}^{-1}\end{array}$ & $\begin{array}{c}B_{v}^{\prime}(\text { calc }) \\
\mathrm{cm}^{-1}\end{array}$ & Footnotes \\
\hline 0 & 63704.85 & 63704.85 & 1.2748 & 1.2748 & (a) \\
\hline 1 & ${ }^{\mathrm{e}} 64802.74$ & 64801.55 & e 1.2582 & 1.2573 & (a) \\
\hline 2 & 65877.91 & 65877.79 & 1.2403 & 1.2399 & (c) \\
\hline 3 & 66933.75 & 66934.05 & 1.2225 & 1.2225 & (c) \\
\hline 4 & 67970.84 & 67970.71 & 1.2048 & 1.2053 & (c) \\
\hline 5 & & 68988.08 & & 1.1882 & \\
\hline 6 & 69986.64 & 69986.41 & 1.1712 & 1.1712 & (a) \\
\hline 7 & 70965.85 & 70965.88 & 1.1540 & 1.1544 & (a) \\
\hline 8 & 71926.47 & 71926.63 & e 1.1360 & 1.1377 & (b) \\
\hline 9 & & 72868.75 & & 1.1210 & \\
\hline 10 & 73792.28 & 73792.29 & 1.1052 & 1.1045 & (b) \\
\hline 11 & & 74697.27 & & 1.0880 & \\
\hline 12 & 75583.55 & 75583.66 & e 1.0636 & 1.0714 & (a) \\
\hline 13 & & 76451.39 & & 1.0549 & \\
\hline 14 & 77300.59 & 77300.35 & 1.0404 & 1.0382 & (a) \\
\hline 15 & 78130.39 & 78130.37 & 1.0198 & 1.0214 & (a) \\
\hline 16 & & 78941.22 & & 1.0045 & \\
\hline 17 & 79732.45 & 79732.61 & 0.9857 & 0.9872 & (a) \\
\hline 18 & & 80504.15 & & 0.9695 & \\
\hline 19 & & 81255.38 & & 0.9514 & \\
\hline 20 & & 81985.69 & & 0.9328 & \\
\hline 21 & 82694.39 & 82694.37 & 0.9144 & 0.9135 & (a) \\
\hline 22 & е 83379.8 & 83380.56 & & 0.8934 & (d) \\
\hline 23 & & 84043.20 & & 0.8725 & \\
\hline 24 & 84681.14 & 84681.06 & 0.8511 & 0.8506 & (a) \\
\hline 25 & 85292.60 & 85292.68 & 0.8269 & 0.8275 & (a) \\
\hline 26 & & 85876.34 & & 0.8032 & \\
\hline 27 & 86430.08 & 86430.07 & 0.7775 & 0.7775 & (a) \\
\hline
\end{tabular}

a Data from transitions reported in this paper.

"Data from Herzberg and Hugo [1].

Data for upper state levels of transitions for which line assignments were reported by Herzberg and Hugo [1]. The constants and band origins given here are from measurements and analyses of new high resolution plates.

${ }^{d}$ From observed band head.

e Perturbed band origins and $B_{r}^{\prime}$-values not included in the least squares fit.

because of Coriolis interactions of the $\mathrm{I}$ and $\mathrm{D}$ states with the $A^{1} I I$ state. The $I-X$ bands consist of a single $\mathrm{Q}$-branch and the $\mathrm{D}-\mathrm{X}$ bands consist of $\mathrm{P}, \mathrm{Q}$, and R-branches.
The analyses of two new $I-X$ bands and one new $\mathrm{D}-\mathrm{X}$ band are given in tables 9 and 10 , respectively. A second new band head of the $D-X$ system, $(6-0)$, has also been observed at $1398.0 \AA$, but the band is too overlapped to be thoroughly analyzed.

The vibrational assignment of the new $I-X$ 'bands as (21-0) and (24-0) was obtained by a reasonably short extrapolation of the band origins and $B_{r}^{\prime}$-values observed earlier [4]. The assignment of the (21-0) $\mathrm{D}-\mathrm{X}$ band is somewhat less certain since only three other bands of this transition have been observed, viz, the $(1-0)$ and $(6-0)$ bands for the ${ }^{12} \mathrm{C}^{16} \mathrm{O}$ and the (1-0) band for the ${ }^{13} \mathrm{C}^{16} \mathrm{O}$ isotope. The assignment of the $\mathrm{D}^{1} \Delta-\mathrm{X}^{1} \Sigma^{+}$band at $83644 \mathrm{~cm}^{-1}$ to the $\nu^{\prime}=21$ level is based upon an extrapolation from previously reported data [5] and upon the correlation of the spectroscopic constants of the $\mathrm{D}^{1} \Delta$ state with those of other states from the same electronic configuration.

The values $\omega_{e}, x \omega_{e}$, and $\alpha_{e}$ determined from the three most probable assignments of the levels in question are:

\begin{tabular}{l|c|c|c}
\hline \hline$v^{\prime}$ & 20 & 21 & 22 \\
\hline & & & \\
$\omega_{e}$ & 1074.0 & 1094.0 & 1109.4 \\
$x \omega_{e}$ & 7.69 & 10.20 & 12.11 \\
$\alpha_{e}$ & 0.0175 & 0.0167 & 0.0159 \\
\hline
\end{tabular}

From a comparison of these constants with those of the $d^{3} \Delta_{i}$ state and other states of the same configuration the $v^{\prime}=21$ assignment is the most probable.

Band origins and $B_{v}^{\prime}$-values obtained from the new bands observed here have been combined with similar data from the earlier work to determine revised spectroscopic constants for the $I^{1} \Sigma-$ and $D^{1} \Delta$ states. The band origins and $B_{r}^{\prime}$-values for the two states are given in tables 11 and 12 , respectively. The constants are given in tables 13 and 14, respectively. The constants for the $D^{1} \Delta$ state are necessarily less accurate than those for other systems since so few $\mathrm{D}-\mathrm{X}$ bands have been observed.

TABLE 7. Vibrational and rotational constants for the $\mathrm{e}^{3 \Sigma^{-}-}$state $^{a}$

\begin{tabular}{|c|c|}
\hline $\begin{array}{l}Y_{01}^{(4)}=1.2836 \pm 0.0013 \mathrm{~cm}^{-1} \\
Y_{11}^{(+)}=(-1.7528 \pm 0.0657) \times 10^{-2} \mathrm{~cm}^{-1} \\
Y_{21}^{(4)}=(0.0707 \pm 0.9809) \times 10^{-4} \mathrm{~cm}^{-1}\end{array}$ & $\begin{aligned} B_{e} \cong Y_{01}^{(1,2)} & =1.2836 \pm 0.0024 \mathrm{~cm}^{-1} \\
-\alpha_{e^{\prime}} \cong Y_{11,2)}^{(1,2)} & =-0.0174 \pm 0.0005 \mathrm{~cm}^{-1} \\
T_{100} & =63074.85 \pm 0.30 \mathrm{~cm}^{-1}\end{aligned}$ \\
\hline $\begin{array}{l}Y_{31}^{(+)}=(6.6755 \pm 5.4187) \times 10^{-6} \mathrm{~cm}^{-1} \\
Y_{41}^{(1)}=(-2.9415 \pm 0.9810) \times 10^{-7} \mathrm{~cm}^{-1}\end{array}$ & $\begin{aligned} T_{e}^{(2,3)} & =64230.9 \pm 2.0 \mathrm{~cm}^{-1} \\
\omega_{e} \cong Y_{10}^{(2,3)} & =1116.5 \pm 1.5 \mathrm{~cm}^{-1}\end{aligned}$ \\
\hline $\begin{array}{l}T_{e 0}^{(6)}=63148.65 \pm 0.30 \mathrm{~cm}^{-1} \\
T_{e}^{(6)}=64230.24 \pm 0.30 \mathrm{~cm}^{-1} \\
Y_{10}^{(6)}=1117.72 \pm 0.28 \mathrm{~cm}^{-1} \\
Y_{10}^{(6)}=-10.686 \pm 0.092 \mathrm{~cm}^{-1} \\
Y_{20}^{(6)}=(1.1744 \pm 0.1284) \times 10^{-1} \mathrm{~cm}^{-1} \\
Y_{(0)}^{(6)}=(-5.5044 \pm 0.8598) \times 10^{-3} \mathrm{~cm}^{-1} \\
Y_{50}^{(6)}=(1.5900 \pm 0.2725) \times 10^{-4} \mathrm{~cm}^{-1} \\
Y_{50}^{(6)}=(-2.7042 \pm 0.3278) \times 10^{-6} \mathrm{~cm}^{-1}\end{array}$ & $\begin{aligned}-x \omega_{e} \cong Y^{20} & (2,3) \\
{ }^{20} D^{\prime} & =-9.8 \pm 0.4 \mathrm{~cm}^{-1} \\
I_{e} & =21.806 \times 10^{-6} \mathrm{~cm}^{-1} \\
r_{e} & =1.384 \mathrm{~A}\end{aligned}$ \\
\hline
\end{tabular}


TABLE 8. Observed wave numbers in the $\mathrm{e}^{3} \Sigma^{-}-\mathrm{X}^{1} \Sigma^{+}$system of ${ }^{13} \mathrm{C}^{16} \mathrm{O}$

\begin{tabular}{r|r|r|r}
\hline \multicolumn{4}{|c}{$(2-0)$ Band } \\
\hline$J$ & \multicolumn{1}{c}{${ }^{\circ} \mathbf{P}(\mathrm{J})$} & ${ }^{\mathrm{Q}} \mathbf{Q}(\mathrm{J})^{\mathrm{a}}$ & \multicolumn{1}{c}{${ }^{\mathrm{S}} \mathrm{R}(\mathrm{J})$} \\
\hline 0 & & 65842.06 & 65849.27 \\
1 & & 841.03 & 852.29 \\
2 & 65830.95 & 838.46 & 854.50 \\
3 & ${ }^{*} 822.33$ & $* 834.73$ & 855.33 \\
4 & 812.25 & 829.21 & 854.88 \\
5 & 800.97 & 822.82 & 853.10 \\
6 & & $* 814.98$ & 849.98 \\
7 & & 805.86 & 845.53 \\
8 & & 795.33 & $* 839.73$ \\
9 & & 783.17 & \\
10 & & & 814.98 \\
11 & & & \\
\hline
\end{tabular}

*Blended lines.

a All three Q-type branches unresolved.

TABLE 9. Observed wave numbers in the $\mathrm{I}^{1} \Sigma^{-}-\mathrm{X}^{1} \Sigma^{+}$system of $\mathrm{CO}$

\begin{tabular}{r|r|r}
\hline \hline & $(21-0)$ Band & $(24-0)$ Band \\
\hline$J$ & $Q(J)$ & \multicolumn{1}{c}{$Q(J)$} \\
\hline 7 & & 84542.19 \\
8 & & 524.61 \\
9 & 82680.62 & 504.66 \\
10 & 659.83 & 482.53 \\
11 & $* 637.30$ & 458.09 \\
12 & 611.72 & 431.62 \\
13 & & 371.70 \\
14 & 555.50 & 338.47 \\
15 & 524.15 & \\
16 & 490.16 & \\
\hline
\end{tabular}

*Blended lines.

TABLE 10. Observed wave numbers in the $\mathrm{D}^{1} \Delta-\mathrm{X}^{1} \Sigma+$ system of $\mathrm{CO}$

(21-0) Band

\begin{tabular}{r|r|r|r}
\hline$J$ & \multicolumn{1}{|c|}{$\mathrm{P}(\mathrm{J})$} & $\mathrm{Q}(\mathrm{J})$ & \multicolumn{1}{c}{$\mathrm{R}(\mathrm{J})$} \\
\hline & & $* 83643.56$ & \\
3 & & $* 635.16$ & $* 83643.56$ \\
4 & & $* 624.79$ & $* 635.16$ \\
5 & 83615.67 & $* 61.33$ & $* 624.79$ \\
6 & 601.98 & $* 612.33$ \\
7 & 585.32 & $* 597.90$ & $* 612.33$ \\
8 & 567.17 & $* 581.34$ & $* 597.90$ \\
9 & 547.07 & 563.23 & $* 581.34$ \\
10 & 524.85 & 542.50 & 562.69 \\
11 & 500.45 & $* 520.07$ & 541.64 \\
12 & 474.01 & 495.40 & 518.73 \\
13 & 445.53 & 468.71 & 493.66 \\
14 & 415.09 & 440.04 & 467.01 \\
15 & & 409.18 & 407.78 \\
16 & & & \\
\hline
\end{tabular}

*Blended lines.
TABLE 11. Band origins and $\mathrm{B}_{\mathrm{v}}^{\prime}$-values for the $\mathrm{I}^{1} \Sigma^{-}-\mathrm{X}^{1} \Sigma^{+}$system

\begin{tabular}{|c|c|c|c|c|}
\hline$v^{\prime}$ & $\begin{array}{c}\nu_{o}(\text { obs }) \\
\mathrm{cm}^{-1}\end{array}$ & $\begin{array}{c}\nu_{o}(\text { calc })^{a} \\
\mathrm{~cm}^{-1}\end{array}$ & $\begin{array}{c}B_{r}^{\prime}(\mathrm{obs}) \\
\mathrm{cm}^{-1}\end{array}$ & $\begin{array}{c}B_{v}^{\prime}(\text { calc })^{a} \\
\mathrm{~cm}^{-1}\end{array}$ \\
\hline 0 & & 64546.25 & & 1.2613 \\
\hline 1 & 65617.32 & 65617.24 & 1.2427 & 1.2434 \\
\hline 2 & 66667.04 & 66667.28 & 1.2268 & 1.2258 \\
\hline 3 & 67696.60 & 67696.65 & & 1.2085 \\
\hline 4 & 68706.08 & 68705.60 & 1.1915 & 1.1915 \\
\hline 5 & 69692.39 & 69694.34 & 1.1748 & 1.1745 \\
\hline 6 & 70662.70 & 70663.05 & 1.1568 & 1.1576 \\
\hline 7 & & 71611.85 & & 1.1406 \\
\hline 8 & & 72540.86 & & 1.1235 \\
\hline 9 & 73450.10 & 73450.12 & 1.1060 & 1.1062 \\
\hline 10 & & 74339.65 & & 1.0888 \\
\hline 11 & & 75209.42 & & 1.0710 \\
\hline 12 & 76059.70 & 76059.33 & 1.0538 & 1.0530 \\
\hline 13 & 76889.00 & 76889.26 & & 1.0347 \\
\hline 14 & & 77699.02 & & 1.0160 \\
\hline 15 & 78488.45 & 78488.37 & 0.9967 & 0.9971 \\
\hline 16 & 79256.90 & 79257.01 & & .9778 \\
\hline 17 & & 80004.59 & & .9582 \\
\hline 18 & & 80730.67 & & .9384 \\
\hline 19 & & 81434.80 & & .9183 \\
\hline 20 & & 82116.40 & & .8979 \\
\hline 21 & 82774.91 & 82774.87 & .8775 & .8775 \\
\hline 22 & & 83409.53 & & .8569 \\
\hline 23 & & 84019.61 & & .8363 \\
\hline 24 & 84604.27 & 84604.28 & .8158 & .8158 \\
\hline
\end{tabular}

TABLE 12. Band origins and $\mathrm{B}_{\mathrm{v}}^{\prime}$-values for the $\mathrm{D}^{1} \Delta-\mathrm{X}^{1} \Sigma^{+}$system

\begin{tabular}{r|c|c}
\hline \hline$v^{\prime}$ & $\begin{array}{c}\nu_{o} \\
\mathrm{~cm}^{-1}\end{array}$ & $\begin{array}{c}B_{r}^{\prime} \\
\mathrm{cm}^{-1}\end{array}$ \\
\hline 1 & $\begin{array}{c}66464.65 \\
a\end{array}$ & 1.2318 \\
6 & $\begin{array}{c}7527 \\
21\end{array}$ & 0.8977 \\
\hline
\end{tabular}

"Band origin estimated from band-head measurement.

\section{Determination of Vibrational and Rotational Constants}

The vibrational and rotational constants were obtained from least squares fits of the observed data to expressions of the following form:

$\nu_{v-0}=T_{e 0}^{(n)}+Y_{10}^{(n)}\left(v+\frac{1}{2}\right)+Y_{20}^{(n)}\left(v+\frac{1}{2}\right)^{2}+\ldots \underset{Y_{n 0}^{(n)}\left(v+\frac{1}{2}\right)^{n}}{+}$ and

$$
Y_{n 0}^{(n)}\left(v+\frac{1}{2}\right)^{n}
$$

$$
B_{v}=Y_{01}^{(m)}+Y_{11}^{(m)}\left(v+\frac{1}{2}\right)+Y_{21}^{(m)}\left(v+\frac{1}{2}\right)^{2}+\underset{Y_{m 1}^{(m)}\left(v+\frac{1}{2}\right)^{m},}{. .+},
$$

where $n$ and $m$ are labels on the coefficients to indicate the order of the power series in $\left(v+\frac{1}{2}\right)$ used to fit the observed band origins $\nu_{v-0}$ and rotational constants $B_{v}$, respectively. $T_{e_{0}}$ is the energy separation between 
TABLE 13. Vibrational and rotational constants for the $\mathrm{I}^{1} \Sigma^{-}$state $^{\text {a }}$

\begin{tabular}{l|r}
\hline \hline$Y_{01}^{(4)}=1.2705 \pm 0.0014 \mathrm{~cm}^{-1}$ & $B_{e} \cong Y_{01}^{(1,2)}=1.2688 \pm 0.0014 \mathrm{~cm}^{-1}$ \\
$Y_{11}^{(4)}=(-1.8480 \pm 0.0762) \times 10^{-2} \mathrm{~cm}^{-1}$ & $-\alpha_{e} \cong Y_{11}^{(1,2)}=-0.0170 \pm 0.0008 \mathrm{~cm}^{-1}$ \\
$Y_{21}^{(4)}=(2.9114 \pm 1.2118) \times 10^{-4} \mathrm{~cm}^{-1}$ & $T_{00}=64546.2 \pm 1.3 \mathrm{~cm}^{-1}(\mathrm{calc})$ \\
$Y_{31}^{(4)}=(-2.0925 \pm 0.7242) \times 10^{-5} \mathrm{~cm}^{-1}$ & $T_{\mathrm{e}}^{(2,3)}=65086.6 \pm 2.5 \mathrm{~cm}^{-1}$ \\
$Y_{41}^{(4)}=(3.6358 \pm 1.4306) \times 10^{-7} \mathrm{~cm}^{-1}$ & $\omega_{e} \cong Y^{(2,3)}=-1090.0 \pm 2.1 \mathrm{~cm}^{-1}$ \\
$T_{e 0}^{(5)}=64002.81 \pm 0.96 \mathrm{~cm}^{-1}$ & $-x \omega_{\mathrm{e}} \cong Y_{20}^{(2,3)}=-10.1 \pm 0.15 \mathrm{~cm}^{-1}$ \\
$T_{e}^{(5)}=65084.40 \pm 0.96 \mathrm{~cm}^{-1}$ & ${ }^{\mathrm{b}} D=9.0 \times 10^{-6} \mathrm{~cm}^{-1}$ \\
$T_{10}^{(5)}=1092.218 \pm 0.69 \mathrm{~cm}^{-1}$ & $I_{e}=22.061 \times 10^{-40} \mathrm{~g} \mathrm{~cm}^{2}$ \\
$Y_{20}^{(5)}=-10.7043 \pm 0.1572 \mathrm{~cm}^{-1}$ & $r_{e}=1.392 \AA$ \\
$Y_{30}^{(5)}=(5.5376 \pm 1.5139) \times 10^{-2} \mathrm{~cm}^{-1}$ & \\
$Y_{40}^{(5)}=(-1.0798 \pm 0.6418) \times 10^{-3} \mathrm{~cm}^{-1}$ & \\
$Y_{50}^{(5)}=(-1.7895 \pm 0.9896) \times 10^{-5} \mathrm{~cm}^{-1}$ & \\
\hline \multicolumn{2}{|c}{ a See footnotes of table 3. } \\
b Used as constant for all levels (determined from the analysis of the 2-0 band).
\end{tabular}

TABLE 14. Vibrational and rotational constants for the $\mathrm{D}^{1} \Delta$ state

\begin{tabular}{rr}
\hline \hline$B_{e} \cong Y_{01}$ & $=1.257 \mathrm{~cm}^{-1}$ \\
$-\alpha_{e} \cong Y_{11}$ & $=-0.017 \mathrm{~cm}^{-1} \quad \omega_{e} \cong Y_{10}=1094.0 \mathrm{~cm}^{-1}$ \\
$T_{00}=65391 \mathrm{~cm}^{-1}$ & \\
\hline
\end{tabular}

the $v=0, J=0$ energy level of the ground electronic state, $\mathrm{X}^{1} \Sigma^{+}$, and the minimum of the potential energy curve of the excited electronic state. $T_{e}$ is obtained by adding the zero-point energy of the $\mathrm{X}^{1} \Sigma^{+}$state to $T_{e 0}$.

Traditionally, spectroscopic constants would be determined from the empirical fit of all the observed data to the above expressions. The coefficients obtained would be the Dunham coefficients and the equilibrium constants $B_{e}, \omega_{e}$, etc. would be obtained by applying the Dunham corrections to these coefficients. Since the Dunham corrections for a molecule like CO are very small, one would normally set the first few Dunham coefficients approximately equal to the equilibrium constants (for example, $B_{e} \cong Y_{01}$ ).

We have not followed the traditional procedure here for the $a^{\prime}, e$, and I states in CO for reasons discussed now. For these states the observed data extend over such a large portion of the potential energy curve that relatively high order expressions are required to fit the observed data. The coefficients of the high order expressions, however, vary considerably as a function of both the order of the expression used and the number of data points included in the fit. Therefore, the first few coefficients of a particular high order least squares fit including all the observed data do not necessarily represent the best values for the equilibrium constants.

The best equilibrium constants can be determined by fitting the observed data for the lower vibrational levels to relatively low order expressions. The disadvantage of quoting only these values for the spectroscopic constants is that they do not accurately reproduce the observed data for the higher vibrational levels. We have, therefore, chosen to report here two sets of constants for the states mentioned above. (1) A high order set of coefficients, which taken as a set reproduce the data over the entire observed range and which further: (a) predict rather accurately any missing data points within the range of observed data, even at high vibrational levels, and (b) give a smoothed polynomial for all the observed data from which potential curves and subsequently Franck-Condon factors can be calculated. (2) A set of low order coefficients which are individually meaningful within stated error limits and which best correspond to the traditional equilibrium spectroscopic constants.

The set of equilibrium constants were determined in the following way. First, the coefficients were obtained for two or more low orders as a function of the number of data points included in the fit. For example, the $B_{r}$-values of the $e^{3 \Sigma}-$ state were fit to expressions of first and second orders. For each order the least squares fit is performed repeatedly, successively excluding the highest- $v \quad B_{r}$-value of the previous fit until the number of $B_{r}$-values included is just greater than the number of coefficients in the expression. Then the values of a particular coefficient, for example, $Y_{01}$, are plotted against the number of vibrational levels included in the fits for the two orders. Such a graph is illustrated in figure 1. The error bars indicated on the plotted values represent one standard deviation as given by the least squares subroutine ORTHO. The quoted value for the equilibrium constant is then determined visually from the graph and the size of the error limits chosen to enclose most of the values in the stable range of the coefficient. Therefore, the value quoted is relatively independent of the order of the expression and the number of data points included in the fits.

The set of high order coefficients is that obtained from a single least squares fit of the observed data to expressions of the particular order indicated by the superscript on the coefficients. The limits given in the tables for these coefficients are one standard deviation. The values of an individual coefficient, particularly. those of the higher terms, are very much dependent on the order of the expression and the number of data points included in the fit. For this reason the individual coefficients are rather meaningless, but the set taken as a whole is significant to the extent that it gives a smoothed polynomial that accurately represents the data over the entire observed range. These polynomials are very useful for interpolation, but it would be very 


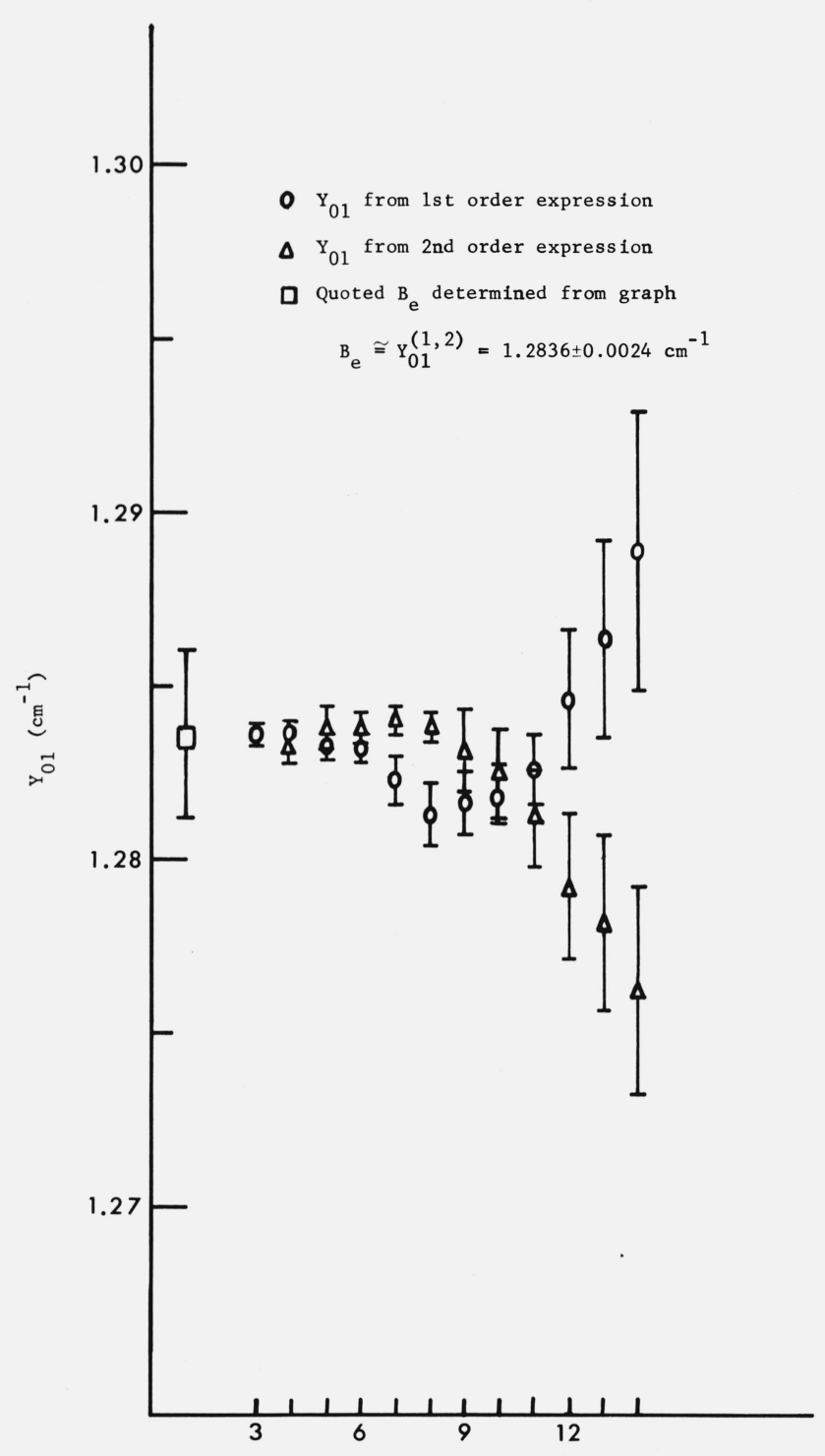

Number of vibrational levels included in the fit

FIGURE 1. Graphical display of $\mathrm{Y}_{01}$-values for the $\mathrm{e}^{3} \Sigma^{-}$state determined from least squares fits of the $\mathrm{B}_{v}$-values to first and second order expressions as a function of the number of $\mathrm{B}_{v}$-values included in the fit. The error bars on the $\mathrm{Y}_{01}$, circles and triangles, indicate one standard deviation as given by the NBS least squares subroutine ORTHO. The equilibrium constant $\mathrm{B}_{e} \cong \mathrm{Y}_{01}^{(1,2)}$ and its associated error limits were determined visually from the graph. dangerous to extrapolate them outside the range of observed data.

We are greatly indebted to G. Herzberg, A. M. Bass, and F. Alberti for their help in obtaining the plates at the National Research Council of Canada and to R. Naber for his help with the experimental work at the U.S. Naval Research Laboratory.

\section{References}

[1] Herzberg, G., and Hugo, T. J., Can. J. Phys. 33, 157 (1955).

[2] Krupenie, P. H., The Band Spectrum of Carbon Monoxide, Nat. Stand. Ref. Data Ser., Nat. Bur. Stand. (U.S.), 5, 92 pages (July 1966).

[3] Stepanov, B. I., J. Phys. U.R.S.S. 2, 205 (1940).

[4] Herzberg, G., Simmons, J. D., Bass, A. M., and Tilford, S. G., Can. J. Phys. 44, 3039 (1966).

[5] Simmons, J. D., and Tilford, S. G., J. Chem. Phys. 45, 2965 (1966).

[6] Douglas, A. E., and Potter, J. G., Appl. Opt. 1, 727 (1962).

[7] Brix, P., and Herzberg, G., Can. J. Phys. 32, 110 (1954).

[8] Wilkinson, P. G., and Byram, E. T., Appl. Opt. 5, 581 (1965).

[9] Simmons, J. D., Bass, A. M., and Tilford, S. G., Astrophys. J. 155, 345 (1969).

[10] Herzberg, G., Hugo, T. J., Tilford, S. G., and Simmons, J. D., Can. J. Phys. 48, 3004 (1970).

[11] Slanger, T. G., and Black, G., in press.

[12] Gero, L. Z. Physik 109, 216 (1938).

[13] (,ar, S. N., Indian J. Phys. 23, 161 (1949).

[14] (;aydon, A. G., Dissociation Energies and Spectra of Diatomic Molecules, Third Edition (Chapman and Hall, London, 1968), p. 211. 